\title{
Knowledge of Dental Avulsion Among Emergency Physicians: A Survey Study
}

\author{
๑ Seçil Çalışkan1, ๑ Ebru Delikan², ® Sena Kızılaslan1, ๑ Özge Özbek1 \\ ${ }^{1}$ Eskişehir Osmangazi University Faculty of Dentistry, Department of Pediatric Dentistry, Eskişehir, Turkey \\ ${ }^{2}$ Nuh Naci Yazgan University Faculty of Dentistry, Department of Pediatric Dentistry, Kayseri, Turkey
}

\begin{abstract}
Aim: Dentoalveolar injuries are complex conditions affecting teeth and surrounding tissues and are frequently seen in children or adolescents. The purpose of this study was to investigate the levels of knowledge on dental avulsion among emergency physicians (EPS).

Materials and Methods: A questionnaire was used to investigate EPs' level of knowledge and therapeutic approaches to avulsion. One hundred EPs were included in the study. The questionnaire was mailed to participants, and the data obtained were evaluated. Descriptive statistics and chi-square tests were used for statistical analysis.

Results: Our results showed that although EPs frequently encountered dental traumas, their medical education had not included dental emergencies. The knowledge level of dental avulsion of all EPs included in the study was as follows; $24 \%$ low, $56 \%$ moderate and only $18 \%$ high .

Conclusion: EPs possessed inadequate knowledge concerning the immediate treatment of dental avulsion. The treatment of avulsion should be included in the medical curriculum. This will result in appropriate emergency treatments in dental avulsion being provided in emergency settings.
\end{abstract}

Keywords: Avulsion, dentistry, emergencies, knowledge

\section{Introduction}

Traumatic dental injuries resulting from home/school/ playground accidents, sports activities, fights, or traffic accidents are a serious oral health problem. Although they can be seen during any period of life, such injuries are more common in infancy, childhood and adolescence (1). Dental avulsion, defined as the complete displacement of the tooth from its socket, is one of the most severe traumatic dentoalveolar injuries. According to the American Academy of Pediatric Dentistry, avulsion is one of the most common traumatic dental injuries seen in children (2), and is usually experienced by children aged $2-4$ and $8-12$ years (3). The frequency of dental avulsion varies between $0.5 \%$ and $16 \%$ in permanent dentition and between $7 \%$ and $13 \%$ in primary dentition. These injuries usually affect the incisors, especially the maxillary central incisors (4).

All traumatic dental injuries including avulsion should be considered as emergencies and treated promptly and effectively. In addition to cosmetic difficulties, phonetic, chewing and psychological problems are also encountered following the loss of these teeth (5). Immediate and appropriate replantation of an avulsed permanent tooth is therefore highly important for good long-term prognosis (5). 
When immediate replantation is not possible, appropriate storage media and extra-alveolar time before replantation are important for the success of the treatment. In such injuries, parents frequently first apply to emergency departments $(6,7)$. It is therefore very important that the emergency physician (EP) should have adequate knowledge concerning the emergency management of dental traumas and that they administer the appropriate treatment immediately.

The aim of the present study was to evaluate knowledge on avulsion among EPs.

\section{Materials and Methods}

This study was planned and conducted in line with the ethical standards detailed in the Declaration of Helsinki and approved by the Ethical Committee of Mersin University (2018/173).

This cross-sectional study involved EPs working at university hospitals or public training and research hospitals in Turkey. The inclusion criteria for participants were as follows; working in a hospital as an EP, willingness to take part to the study, and aged $20-60$ years. Participants who agreed to participate in this research with their own consent were included. The authors modified a previously used and validated questionnaire (6). All questions were close-ended (multiple-choice), and the questionnaire was divided into three sections, the first consisting of four questions about the personal characteristics of the participating physicians. The second section included 11 questions evaluating physicians' attitudes to two different hypothetical avulsion cases, and the final section consisted of two questions evaluating self-assessment. A pilot study to evaluate intelligibility was conducted before the main study, and a few modifications were applied. The 17-item questionnaire was administered to the EPs by e-mail. For reasons of privacy, no names or other personal information were recorded. Returning the questionnaire was interpreted as indicating willingness to participate in the study. A total of 90 participants was found to be sufficient (power: 0.95, effect size: 0.3 and 5\% standard error). The questionnaire was e-mailed to 150 EPs to compensate for possible dropouts, and 100 EPs finally participated in the study.

Knowledge levels were assessed using a standardized scoring method modified from Abu-Dawoud et al. (8). Eleven questions from the second section of the questionnaire were used to evaluate knowledge levels. A score of 8 indicated full knowledge while a score of 0 indicated no knowledge. Other mean scores were formulated as 6-8 points indicating high knowledge, 3-5 points indicating moderate knowledge, and 0-2 points indicating low knowledge.

\section{Statistical Analysis}

Statistical Analysis was performed on IBM SPSS 22 (IBM Corp, Armonk, NY) software. Descriptive statistics were used for the evaluation of the data. Chi-square tests were used to analyze relationships between age, gender, previous education concerning dental trauma, and knowledge levels regarding the emergency management of dental trauma. Statistical significance was set at $p<0.05$ for all tests.

\section{Results}

One hundred EPs participated in the study (34\% female and $66 \%$ male). Nearly half (46\%) were aged $31-40$ years, and the mean length of professional experience was 9.5 years. Approximately half of the participants (52\%) reported that their education had not included information about the management of dental trauma. The majority of physicians $(80 \%)$ also stated that they had never encountered a dental injury (Table I).

When physicians were classified according to their levels of dental trauma knowledge, $24 \%$ had a low level of knowledge, $56 \%$ had moderate knowledge and only $18 \%$ had high knowledge. The chi-square test results revealed

Table I. Demographic characteristics of the EP

\begin{tabular}{|c|c|}
\hline & (\%) \\
\hline \multicolumn{2}{|l|}{ Gender } \\
\hline Female & 34 \\
\hline Male & 66 \\
\hline \multicolumn{2}{|l|}{ Age } \\
\hline $18-30$ & 32 \\
\hline $31-40$ & 46 \\
\hline$>40$ & 22 \\
\hline \multicolumn{2}{|c|}{ Years of experience } \\
\hline$<5$ & 31 \\
\hline $5-10$ & 42 \\
\hline$>10$ & 27 \\
\hline \multicolumn{2}{|c|}{ Education on management of dental trauma } \\
\hline Yes & 48 \\
\hline No & 52 \\
\hline \multicolumn{2}{|c|}{ Eye-witnessed a dental injury } \\
\hline Yes & 20 \\
\hline No & 80 \\
\hline
\end{tabular}


no statistically significant relationships between levels of knowledge concerning avulsed teeth and the physician's age, gender, experience, or previous education regarding dental trauma.

In the hypothetical permanent tooth avulsion case, $78 \%$ of EPs were able to accurately identify the tooth concerned. Unfortunately, however, $72 \%$ of physicians did not suggest replantation. The majority of participants (68\%) stated that the avulsed tooth should be held without touching the root. The great majority (84\%) agreed that the tooth should be cleaned before replantation, the most popular method being to clean it under tap water (54\%). Tetanus vaccination was also described as being necessary by the great majority (80\%). In cases when the avulsed tooth could not be replanted, the most popular storage media among the physicians were milk (28\%), water $(16 \%)$, or a handkerchief (14\%). Regrettably, only $28 \%$ of the emergency specialists correctly answered the question concerning the replantation time of the avulsed tooth, a factor which is very important in prognosis (Table II).

Table II. Knowledge of EP

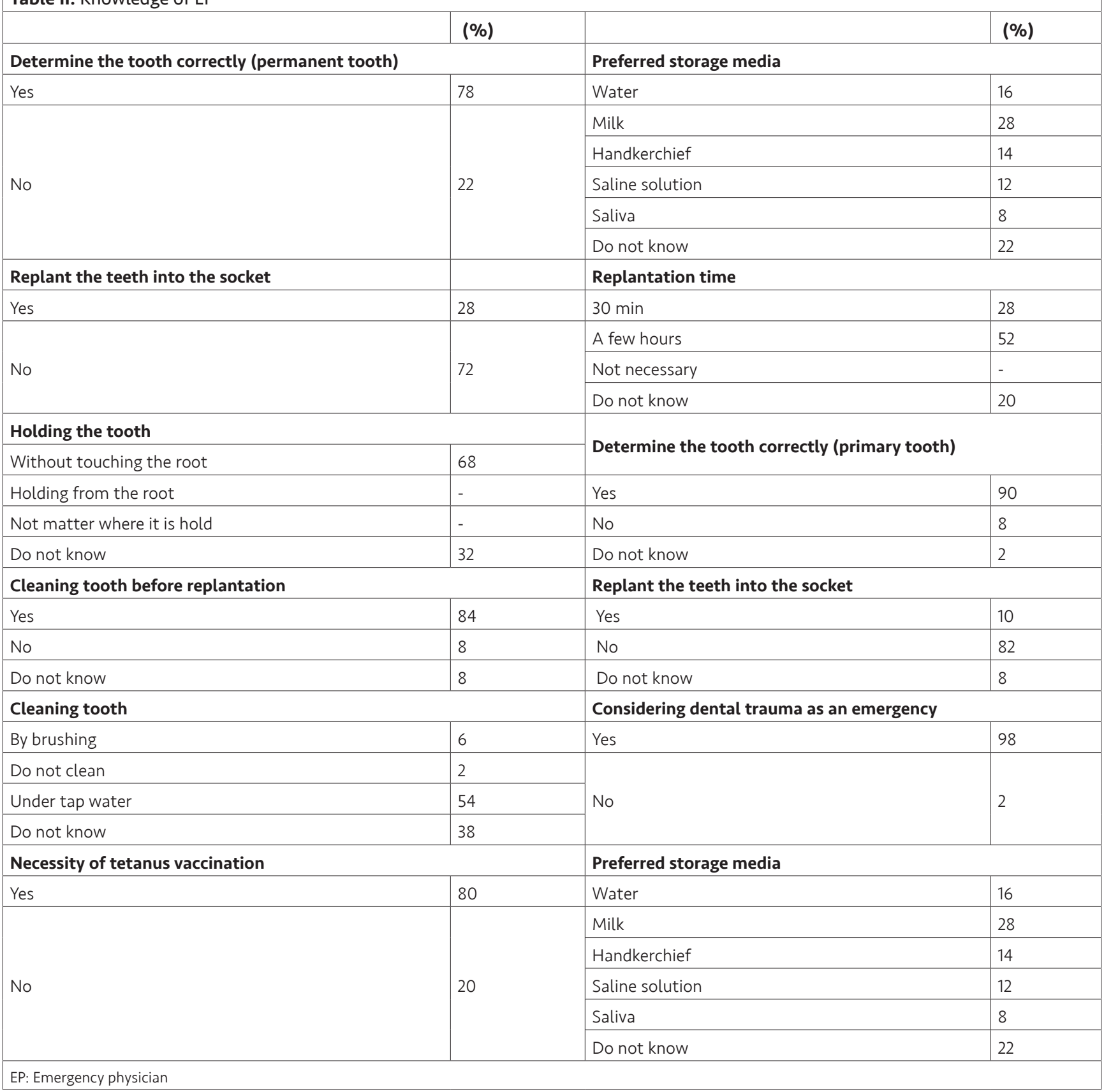


In the hypothetical primary tooth avulsion case, the great majority of EPs (90\%) were able to accurately identify the tooth. Most of them (82\%) stated that they would not replant the primary tooth into the socket (Table II). Dental trauma was considered an emergency condition by $98 \%$ of the physicians.

In the third part of the questionnaire, $46 \%$ of physicians reported that they would not intervene in a dental trauma case concerning an avulsed tooth and would refer the patient to a dentist. Almost all (90\%) EPs reported a need for more training on dental trauma and its management.

\section{Discussion}

Traumatic dental injuries can occur at any age but are particularly common in childhood (9). These injuries may range from a simple enamel fracture to an extensive maxillofacial trauma. In cases of avulsion, the most serious type of injury, families frequently present to emergency services (7). The immediate management provided by EPs greatly affects the prognosis of the tooth $(5,6)$. However, patients may not always be treated appropriately due to a lack of information among EPs regarding dental trauma management. The level of in-time and correct treatment for dental trauma patients was stated to be only $19.29 \%$ in a study conducted by Kayıllıoğlu Zencircioğlu et al. (7) It is therefore very important for EPs to possess sufficient knowledge of dental injury management, as they may be required to perform first-aid in traumatic events. The present study was intended to determine the baseline knowledge levels of EPs regarding dental avulsion management in both primary and permanent dentition.

In previous studies, there was no significant relationship between the gender of the physician and dental trauma knowledge $(6,10,11)$. Bahammam (6) observed a statistically significant relationship between dental trauma knowledge levels and age. In the present study, no significant relationship was found between the age of the EP and their dental trauma knowledge. We originally expected knowledge to increase with age due to increasing professional experience. Unexpectedly, there was no correlation between experience and knowledge level due to the fact that EPs are not trained on dental trauma during their medical education or throughout their working life in Turkey.

Consistent with Lin et al. (10) and Trivedy et al. (12), nearly half of the EPs reported not receiving adequate information about emergency management in dental trauma during or after training. Similarly, lyer et al. (13) reported that 33.3\% of physicians received no training regarding first aid in the area of dental avulsion. In contrast, Needleman et al. (14) reported that the majority of physicians (80.4\%) had received training concerning the management of traumatic dental injuries in Massachusetts emergency departments. This high level was attributed to these physicians being certified in pediatric emergency medicine, and therefore cannot be generalized to all EPs.

Our findings showed that most EPs possessed a moderate knowledge on dental avulsion. Another previous study also reported that EPs possessed moderate knowledge and awareness of dental avulsion (15). It is important to increase EPs' awareness of traumatic dental injuries since they may be the first specialists to encounter dental injuries in emergency departments. Their education should therefore also comprise managing dental trauma. A formal protocol for managing dental injuries and avulsed teeth should also be established for emergency departments.

Only $20 \%$ of physicians had previously witnessed any dental trauma. In contrast, Raoof et al. (15) reported that $75 \%$ of medical students had witnessed dental trauma. The results of a different study also conducted in Turkey showed that $41.2 \%$ of dental injuries were first examined by EPs, $25 \%$ by physicians, and only $7.3 \%$ by a dentist (11). That study also indicated that EPs had low levels of knowledge regarding the diagnosis and treatment of dental injuries despite having experienced or witnessed such cases (11). EPs are often the first professional to provide emergency management of dental injuries $(7,16)$. These studies demonstrate the importance of dental emergency management being included in the education curriculum.

Similar to the present study, questions also in another in another study about permanent teeth were more often answered correctly than those regarding primary dentition (13). This may be explained by EPs having difficulty in distinguishing primary and permanent teeth and by their being more familiar with permanent dentition.

Immediate replantation is important for the prognosis of avulsed teeth (6). However, nearly half of the EPs in this study (48\%) reported being unaware of what to do when encountering an avulsed tooth. More than half of the participants $(54 \%)$ stated that they would prefer to communicate with a dental office in cases of such injuries. Other studies also reported that most participants would prefer to contact a dentist $(6,10)$. This may be due to participants lacking knowledge and confidence in terms of providing first aid in cases of traumatic dental injuries. Another problem to note is that dental trauma is not considered an emergency in hospital emergency departments. 
The majority of participants in the present study stated that the permanent avulsed tooth should be replanted. This result was consistent with the results of the study of lyer et al. (13), but not with the results of the study of Holan and Shmueli (1), who reported that only $4 \%$ of physicians would replant the permanent tooth under any circumstances. However, half of the participants stated that they would not replant the avulsed permanent tooth under any circumstances. This indicates the low awareness among physicians of the possibility of saving the tooth through simple replacement in the socket. In addition, only $5 \%$ of participants in another study were aware of replantation (17). The main reason for this low rate may be the participants were physicians working in medical colleges or as residents in advanced medical training programs, and were therefore less familiar with emergency situations than EPs.

The avulsed tooth should not be held by the root. Touching the root is contraindicated as this may damage the remaining periodontal tissue and increase contamination of the avulsed tooth (14). In this study, the participants' knowledge regarding holding the avulsed tooth without touching the root was highly accurate. This was also consistent with several other studies involving $\operatorname{EPs}(8,9,18)$. However, the participants were generally unsure whether they would be able to replant the tooth in the correct position, although they were aware that the avulsed tooth should be cleaned before replanting. Furthermore, the majority of the participants stated that they would remove any debris under tap water. Likewise, almost half the physicians in Bahammam's study recommended rinsing the tooth under running water (6). Similarly, Raoof et al. (15) reported that most of the physicians would rinse the avulsed tooth under tap water without rubbing it.

If the tooth cannot be replanted, it should be placed in a suitable medium such as saliva, milk, blood, plain water, or contact lens solution, or it can also be placed inside the mouth. Theoretically, the best storage media are HBSS, Via Span and Eagle's medium. Our participants generally possessed inadequate knowledge of transport media. The periodontal ligament (PDL) cells can maintain their viability in milk for 6 hours during the extraoral period. Only 28 physicians agreed that the tooth could be preserved in milk. Moreover just 10 participants knew that the tooth could be kept in the mouth for storage. Although the tooth can be conserved in the buccal sulcus in children, there is a risk of the avulsed tooth being swallowed. Conserving the tooth in a handkerchief in a dry environment causes the destruction of PDL cells. This is, therefore, one of the least desirable means of preserving an avulsed tooth. Conserving the tooth in a handkerchief was a less popular choice among our participants compared with a previous study (8). This may be due to the physicians in that study not working in an emergency clinic.

Another factor affecting prognosis and the success of treatment is the extraoral time. After a dry time of $60 \mathrm{~min}$ or more, all PDL cells become non-viable (19). It is therefore important that reimplantation be performed in the first hour. Our participants' knowledge concerning the correct intervention time for an avulsed tooth was surprising. More than half of the participants stated that an avulsed tooth can be treated within a few hours. Only $6 \%$ stated that they would intervene immediately. Our results were in contrast with those of several studies in which physicians were aware of the need for an avulsed permanent tooth to be treated immediately $(6,17)$. In the study of Bahamman et al. (6), there was no "within a few hours" option. The available alternatives were "immediately", "the next day", "within a few days" and "when a symptom developed". Participants who thought that avulsion required intervention on the same day therefore selected the "immediately" option.

In the second hypothetical case involving avulsion of a primary tooth, the majority of physicians were aware that a three-year-old child will have primary dentition. In this situation, approximately half the participants selected informing the parents, instead of referring the patient to a dentist. Moreover, the majority of physicians stated that a primary tooth should not be replanted. The International Association of Dental Traumatology does not recommend replantation of avulsed primary teeth due to the risk of permanent tooth damage (2).

Especially in cases such as avulsion, pediatric patients should be referred to a physician for evaluation of the need for a tetanus booster if the avulsed tooth has come into contact with soil or if tetanus coverage is uncertain (18). In parallel with this information, many participants considered that tetanus vaccination is necessary for emergency intervention in dental injuries.

More than half the physicians felt capable of intervening if they were to encounter a dental trauma. This rate was significantly higher than in Subhashraj (17) study, in which only nine physicians out of 200 were satisfied with their present knowledge on the management of dental injuries. Another study evaluated the confidence levels of physicians in four common dentofacial emergencies, and reported that physicians in emergency departments had the lowest confidence levels in terms of managing dental avulsion injuries (12). Nevertheless, it seems safe to generalize that 
EPs require more education about traumatic dental injuries and their treatment. Our results were in agreement with Ulusoy et al. (20) and Bahammam et al. (6), in which most of the physicians were keen to learn more about dental trauma and felt that they required further education on this subject.

In contrast, a previous study reported that participants working in emergency departments in Massachusetts had received official training regarding traumatic dental injury management and therefore possessed excellent knowledge of managing both permanent and primary tooth avulsions (14). This study shows the need for similar training in Turkey.

\section{Study Limitations}

The main limitations of this study were that it was conducted using a random sampling method and its small sample size. This limitation may have affected the generalization of the study results.

\section{Conclusion}

This study shows a lack of knowledge regarding the immediate treatment of dental avulsions among EPs. Our findings indicate that educational campaigns must be initiated to improve physicians' knowledge regarding the emergency management of traumatic dental injuries.

Local dental organizations should also endeavor to provide the list of dentists who are knowledgeable and willing to be available on a 24-hr basis to the emergency departments. Thus, physicians in emergency departments can consult with these dentists and treat traumatic dental injuries and especially avulsion cases when necessary.

\section{Ethics}

Ethics Committee Approval: The study protocol was approved by the Ethics Committee of Mersin University (2018/173).

Informed Consent: Participants who agreed to participate in this research with their own consent were included.

Peer-review: Externally and internally peer-reviewed.

\section{Authorship Contributions}

Concept: S.Ç., E.D., Design: S.Ç., E.D., Data Collection or Processing: S.Ç., S.K., Analysis or Interpretation: S.Ç., Literature Search: S.Ç., E.D., S.K., Ö.Ö., Manuscript Writing: S.Ç., E.D., S.K.

Conflict of Interest: No conflict of interest was declared by the authors.
Financial Disclosure: The authors declared that this study received no financial support.

\section{References}

1. Holan G, Shmueli Y. Knowledge of physicians in hospital emergency rooms in Israel on their role in cases of avulsion of permanent incisors. International Journal of Paediatric Dentistry 2003; 13:13-9.

2. Council O. Guideline on Management of Acute Dental Trauma. Dental Traumatology 2011; 32:202-12.

3. Nirwan M, Syed AA, Chaturvedi S, Goenka P, Sharma S. Awareness in primary school teachers regarding traumatic dental injuries in children and their emergency management: $\mathrm{A}$ survey in South Jaipur. Int I Clin Pediatr Dent 2016; 9:62-6.

4. Siqueira MBLD, Gomes MC, Oliveira AC, Martins CC, GranvilleGarcia AF, Paiva SM. Predisposing factors for traumatic dental injury in primary teeth and seeking of post-trauma care. Braz Dent I 2013; 24:647-54.

5. Andreasen JO, Andreasen FM, Andersson L. Textbook and color atlas of traumatic injuries to the teeth: John Wiley \& Sons;2018.

6. Bahammam LA. Knowledge and attitude of emergency physician about the emergency management of tooth avulsion. BMC Oral Health 2018; 18:57.

7. Kayıllıoğlu Zencircioğlu Ö, Eden E, Öcek ZA. Access to health care after dental trauma in children: A quantitative and qualitative evaluation. Dental Traumatol 2019; 35:163-70.

8. Abu-Dawoud M, Al-Enezi B, Andersson L. Knowledge of emergency management of avulsed teeth among young physicians and dentists. Dental Traumatol 2007; 23:348-55.

9. Fakhruddin KS, Lawrence HP, Kenny DJ, Locker D. Etiology and environment of dental injuries in 12-to 14-year-old Ontario schoolchildren. Dental Traumatol 2008; 24:305-8.

10. Lin S, Levin L, Emodi O, Fuss Z, Peled M. Physician and emergency medical technicians' knowledge and experience regarding dental trauma. Dental Traumatol 2006; 22:124-6.

11. Yigit Y, Helvacioglu-Yigit D, Kan B, Ilgen C, Yilmaz S. Dentofacial traumatic injuries: A survey of knowledge and attitudes among emergency medicine physicians in Turkey. Dental Traumatol $2019 ; 35: 20-6$

12. Trivedy C, Kodate N, Ross A, et al. The attitudes and awareness of emergency department (ED) physicians towards the management of common dentofacial emergencies. Dental Traumatol 2012; 28:121-6.

13. Iyer SS, Panigrahi A, Sharma S. Knowledge and awareness of first aid of avulsed tooth among physicians and nurses of hospital emergency department. I Pharm Bioallied Sci 2017; 9:94-8.

14. Needleman HL, Stucenski K, Forbes PW, Chen Q, Stack AM. Massachusetts emergency departments' resources and physicians' knowledge of management of traumatic dental injuries. Dental Traumatol 2013; 29:272-9.

15. Raoof M, Vakilian A, Kakoei S, Manochehrifar $H_{\text {, }}$ Mohammadalizadeh S. Should medical students be educated about dental trauma emergency management? A study of physicians and dentists in Kerman Province, Iran. I Dent Educ 2013; 77:494-501. 
16. Nikolic H, Ivancic NJ, Bakarcic D, Hrvatin S, Jakljevic N. Knowledge about emergency procedure in case of dental trauma among paediatricians in Croatia. Eur / Paediatr Dent 2018; 19:277-81.

17. Subhashraj K. Awareness of management of dental trauma among medical professionals in Pondicherry, India. Dental Traumatol 2009; 25:92-4.

18. Flores $M$, Andersson L, Andreasen JO, et al. Guidelines for the management of traumatic dental injuries I. Fractures and luxations of permanent teeth. Dental Traumatol 2007; 1:66-71.
19. Andersson L, Andreasen JO, Day P, et al. International Association of Dental Traumatology guidelines for the management of traumatic dental injuries: 2. Avulsion of permanent teeth. Dental Traumatol 2012; 28:88-96.

20. Ulusoy $A T$, Önder $H$, Cetin B, Kaya Ş. Knowledge of medical hospital emergency physicians about the first-aid management of traumatic tooth avulsion. Int I Paediatr Dent 2012; 22:211-6. 